\title{
Generalidades del recurso eólico en Costa Rica: caso de estudio de la provincia de Cartago
}

\section{Costa Rica's wind resource assessment: A case study for Cartago province}

Gustavo Murillo-Zumbado', Gustavo Richmond-Navarro², Pedro Casanova-Treto ${ }^{3}$, Julio César Rojas-Gómez ${ }^{4}$

Murillo-Zumbado, G; Richmond-Navarro, G;

Casanova-Treto, P; Rojas-Gómez, J.C. Generalidades del recurso eólico en Costa Rica: caso de estudio de la provincia de Cartago. Tecnología en Marcha. Vol. 34-4 OctubreDiciembre 2021. Pág 130-145.

doi) https://doi.org/10.18845/tm.v34i4.5274

1 Instituto Tecnológico de Costa Rica. Costa Rica. Correo electrónico: gmurillo@tec.ac.cr (D) https://orcid.org/0000-0002-1947-3567

2 Instituto Tecnológico de Costa Rica. Costa Rica. Correo electrónico: grichmond@tec.ac.cr Dhttps://orcid.org/0000-0001-5147-5952

3 Universidad de Costa Rica. Costa Rica. Correo electrónico: pedro.casanova@ucr.ac.cr (D) https://orcid.org/0000-0001-8508-6293

4 Instituto Tecnológico de Costa Rica. Costa Rica. Correo electrónico: jrojas@tec.ac.cr 


\title{
Palabras clave
}

Recurso eólico; intensidad de turbulencia; rosa de los vientos.

\section{Resumen}

La evaluación del recurso eólico es vital para el desarrollo e implementación de sistemas de aprovechamiento eólico para la generación de energía. Costa Rica no posee estudios por zonas de manera específica como se plantea, que permita a los profesionales en el área tomar decisiones en función del potencial y características del viento. Con el presente estudio se brinda por primera vez la caracterización del recurso eólico en la provincia de Cartago. La caracterización se realiza para la capa límite superficial, con datos medidos a $10 \mathrm{~m}$ de altura sobre el nivel del suelo tomando la magnitud de la velocidad y dirección del viento obtenidas de ocho estaciones meteorológicas. Durante la caracterización del recurso eólico se analizaron los datos de forma estadística y usando códigos computacionales que permitieron obtener resultados de la información colectada. Al procesar la información brindada por las estaciones meteorológicas se determina que los promedios de velocidades del viento se encuentran entre los $3 \mathrm{~m} / \mathrm{s}$ y $5 \mathrm{~m} / \mathrm{s}$ a una altura de $10 \mathrm{~m}$ sobre la superficie. Además, la intensidad de turbulencia se registra entre $15 \%$ y $30 \%$ y se determinaron dos periodos bien marcados en cuanto a la magnitud del viento, de noviembre a febrero para intensidades fuertes y septiembre a octubre para intensidades más bajas. Para la provincia de Cartago las direcciones que predominan son las componentes Norte o Este, siendo las dos componentes más recurrentes en los datos analizados.

\section{Keywords}

Wind resource; turbulence intensity; wind rose.

\begin{abstract}
Wind resource assessment is essential for the development and implementation of wind power systems. Costa Rica have no studies by zones in a specific way as proposed, which allows professionals in the area to make decisions based on the potential and characteristics of the wind. This study provides for the first time the characterization of the wind resource in the province of Cartago. The characterization is carried out for the surface boundary layer, with data measured at $10 \mathrm{~m}$ above ground level, taking the magnitude of the wind speed and direction obtained from eight meteorological stations. During the characterization of the wind resource, the data was analyzed statistically and using computational codes that allowed obtaining results from the information collected. When processing the information provided by the meteorological stations, it is determined that the average wind speeds are between $3 \mathrm{~m} / \mathrm{s}$ and $5 \mathrm{~m} / \mathrm{s}$ at a height of $10 \mathrm{~m}$ above the surface. In addition, the intensity of turbulence is recorded between $15 \%$ and $30 \%$ and two well marked periods were determined in terms of the magnitude of the wind, from November to February for strong intensities and September to October for lower intensities. For the province of Cartago, the directions that predominate are the North or East components, being the two most recurrent components in the analyzed data.
\end{abstract}

\section{Introducción}

A nivel global las energías renovables y la eficiencia energética son pilares de la transformación energética y una de las claves para mitigar el efecto del cambio climático. En 2017, el sector eléctrico sumó 167 GW de capacidad energética renovable global, esto es un crecimiento 
del $8,3 \%$ con respecto al año 2016 y una continuidad de las tasas de crecimiento de años anteriores, con una media del 8\% anual desde 2010. Siendo de destacar que, entre el 2008 y el 2017, la capacidad global se duplicó [1].

En el caso de la energía eólica, la capacidad instalada acumulada en el planeta en 2001 fue de 23900 MW y en 2017 alcanzó 539123 MW, representando un aumento de más de 22 veces en un periodo de 16 años [2].

En particular, un país que se ha comprometido a alcanzar una matriz de energía eléctrica 100\% renovable para el año 2030 es Costa Rica [3]. Una nación reconocida en la comunidad internacional por sus políticas de conservación y protección de la biodiversidad y que aspira a la carbono neutralidad para el 2100 [4].

Debido a la crisis del petróleo de la década de los 70's, los países como Costa Rica se vieron forzados a buscar nuevas fuentes de producción energética, los primeros estudios sobre energía eólica se realizan finalizando esa década. En la zona de Tilarán se da la construcción de los primeros parques eólicos en la década de los 90's, posteriormente se desarrolló el parque eólico Tejona, perteneciente al Instituto Costarricense de Electricidad (ICE) y que inició sus operaciones en 2002 [5].

De acuerdo con el Plan Nacional de Desarrollo 2015-2018: "Alberto Cañas Escalante", Costa Rica pretende suplir su demanda de energía mediante una matriz que asegure un suministro óptimo, continuo y promoviendo el uso eficiente de energía, con predominio de fuentes renovables y al menor costo [6].

Para el año 2017, el 99,67\% de la generación bruta anual de energía fue mediante fuentes renovables, siendo 11,49\% energía eólica [7]. Además, considerando que en Costa Rica las tres principales fuentes que conforman la matriz energética son: la hidroeléctrica, la eólica y la geotérmica (en ese orden), una de las ventajas de la energía eólica es que forma un buen complemento de la energía hidroeléctrica, pues los vientos se aceleran cuando baja el caudal de los ríos [8].

Para el correcto aprovechamiento de la energía del viento, son necesarios los mapas de potencial eólico. El primero fue realizado en 1984 [9]. Entre 2008 y 2009 el ICE y la Universidad de Costa Rica emplearon modelos de meso-escala, similar al estudio de [23], para elaborar mapas y archivos con datos del recurso eólico en Costa Rica y hasta 20 km dentro del mar [5], un estudio de 2011 presenta el mapa de velocidad del viento media anual [9], siendo el más reciente de 2017, cuando el Banco Interamericano de Desarrollo (BID) realiza un estudio para manejar e incorporar una mayor variedad de energías renovables en Costa Rica [10].

Considerando este panorama, se puede afirmar que Costa Rica no cuenta con estudios sobre el recurso eólico en regiones específicas del país, a partir de datos de estaciones meteorológicas. Al profundizar en este recurso, se podría revelar un potencial en alguna localidad particular, partiendo de un análisis más detallado, como el desarrollado por [11], que basado en variables como la velocidad y, dirección del viento, evidencia un gran potencial en una región colombiana.

Estudios similares en Reino Unido han permitido identificar las mejores zonas para el uso de turbinas de pequeña escala [12]. En Suecia, mediciones directas de la velocidad del viento, mediante mástiles de $100 \mathrm{~m}$ de altura, se emplean para determinar los sitios adecuados para el emplazamiento de parque eólicos [13]. En Nigeria se han desarrollado estudios del perfil de velocidad de viento, procesando mediciones de velocidad de viento a $10 \mathrm{~m}$ de altura, mediante redes neuronales [14]feed-forward, back-propagation network with different configurations, designed using the Neural Toolbox for MATLAB. The monthly mean daily wind speed data 
monitored at $10 \mathrm{~m}$ above ground level for a period of 20 years (1983-2003. Entre tanto en Japón, se emplean técnicas avanzadas de mecánica de fluidos computacional para predecir el comportamiento del viento sobre terreno complejo [15].

El tema de evaluación del recurso eólico es de interés en localidades alrededor de todo el mundo como Pakistán [16], Burkina Faso [17] y Australia [18]. Siendo de interés incluso en desiertos en China [19] y con técnicas sumamente variadas como mediante satélites con escaterómetro en el mar Mediterráneo [20] o bien técnicas estadísticas en tres dimensiones a partir de datos medidos en tierra, empleadas en Alemania, pero exportables a cualquier otra área en el mundo [21].

Con este panorama, en el presente trabajo se caracteriza el recurso eólico en la provincia de Cartago, Costa Rica, la cual contaba con un 65\% de su territorio cubierto por zonas boscosas, para el año 2005 [22]. Esta caracterización se realiza para la capa límite superficial, con datos medidos a $10 \mathrm{~m}$ de altura sobre el nivel del suelo, siendo el primer estudio en su tipo en Costa Rica.

\section{Metodología}

Descripción de la zona de estudio

Este estudio se concentra en la provincia de Cartago, de las siete provincias en las que se divide Costa Rica, Cartago es la segunda más pequeña con área aproximada de 3124,67 km2 y se encuentra ubicada en la parte central del país, como se muestra en la figura 1. Está formada por ocho cantones: Cartago, Paraíso, La Unión, Jiménez, Turrialba, Alvarado, Oreamuno y El Guarco [24].

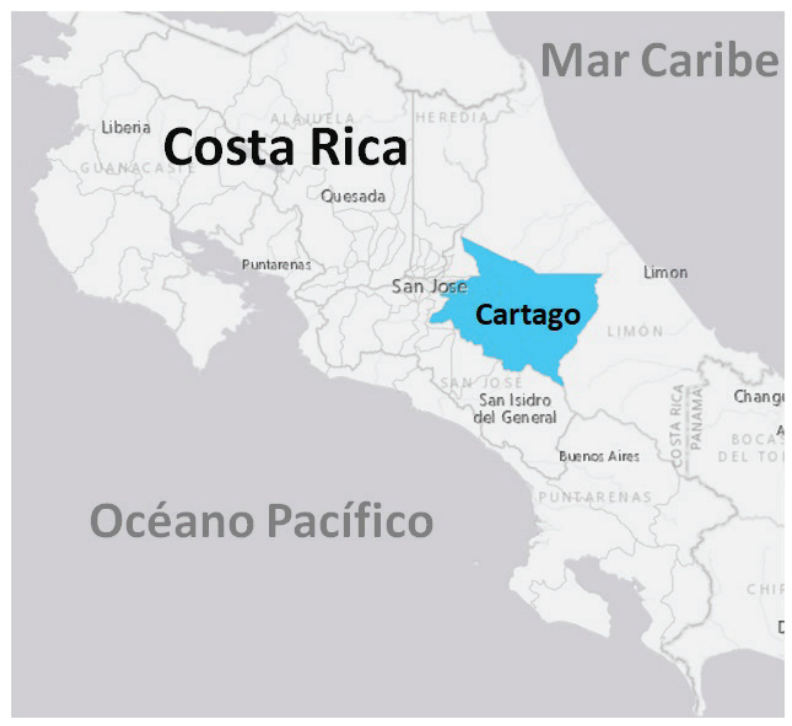

Figura 1. Ubicación geográfica de la provincia de Cartago. Fuente: Atlas de cantones de Costa Rica.

Según su clima, se ubica en la región climática del Valle Central. La región del valle central es afectada por las vertientes del Pacífico y del Caribe, esto genera un clima mixto que permite subdividir la región en oriental y occidental. Cartago se ubica en la parte oriental, zona con mayor influencia de la vertiente del Caribe [25]. La lluvia media anual presente en el valle central va de $1950 \mathrm{~mm}$ a $2820 \mathrm{~mm}$ y la temperatura media anual se encuentra en el rango de $15^{\circ} \mathrm{C}$ a $22{ }^{\circ} \mathrm{C}[26]$. 


\section{Descripción de los datos}

Los datos disponibles son magnitud de la velocidad y dirección del viento. Provienen de ocho estaciones meteorológicas, cuyos detalles se presentan en el cuadro 1 y su ubicación en la Figura 2. Las estaciones ITCR Cartago, RECOPE Ochomogo y Volcán Irazú son administradas por el Instituto Meteorológico Nacional de Costa Rica (IMN), suministran datos con resolución temporal horaria y abarcan un periodo de 10 años de mediciones. Las estaciones Campamento, Terrena Guatuso, San Pablo y Tierra Blanca son administradas por el Instituto Costarricense de Electricidad (ICE), presentan datos con resolución temporal diaria y comprenden un período de 5 años. Por último, la estación meteorológica ITCR CIVCO administrada por el Centro de Investigación en Vivienda y Construcción (CIVCO), con iguales coordenadas de ubicación de la estación ITCR.

Cuadro 1. Estaciones meteorológicas del IMN en la provincia de Cartago.

\begin{tabular}{|c|c|c|c|c|c|}
\hline \multirow[t]{2}{*}{ ID } & \multirow[t]{2}{*}{ Nombre } & \multirow[t]{2}{*}{ Latitud Norte } & \multirow[t]{2}{*}{ Longitud Oeste } & \multicolumn{2}{|c|}{ Periodo } \\
\hline & & & & Inicio & Fin \\
\hline 1 & ITCR Cartago & $09^{\circ} 51^{\prime} 08^{\prime \prime}$ & $83^{\circ} 54^{\prime} 31^{\prime \prime}$ & 01/01/2007 & $31 / 12 / 2017$ \\
\hline 1 & ITCR CIVCO & $09^{\circ} 51^{\prime} 08^{\prime \prime}$ & $83^{\circ} 54^{\prime} 31^{\prime \prime}$ & $31 / 08 / 2011$ & 02/05/2017 \\
\hline 2 & RECOPE Ochomogo & $09^{\circ} 53^{\prime} 40,21^{\prime \prime}$ & $83^{\circ} 56^{\prime} 19,41^{\prime \prime}$ & 01/01/2007 & $31 / 12 / 2017$ \\
\hline 3 & Volcán Irazú & $09^{\circ} 58^{\prime} 47,53^{\prime \prime}$ & $83^{\circ} 50^{\prime} 16,14^{\prime \prime}$ & 01/01/2007 & $31 / 12 / 2017$ \\
\hline 4 & Campamento & $09^{\circ} 52^{\prime} 52,65^{\prime \prime}$ & $83^{\circ} 38^{\prime} 46,28^{\prime \prime}$ & 01/01/2013 & $31 / 12 / 2017$ \\
\hline 5 & Terrena de Guatuso & $09^{\circ} 49^{\prime} 18,90^{\prime \prime}$ & $83^{\circ} 56^{\prime} 37,63^{\prime \prime}$ & $01 / 01 / 2013$ & $31 / 12 / 2017$ \\
\hline 6 & San Pablo & $09^{\circ} 56^{\prime} 14,34^{\prime \prime}$ & $83^{\circ} 50^{\prime} 06,30^{\prime \prime}$ & 01/01/2013 & $31 / 12 / 2017$ \\
\hline 7 & Tierra Blanca & $09^{\circ} 54^{\prime} 47,16^{\prime \prime}$ & $83^{\circ} 53^{\prime} 12,85^{\prime \prime}$ & 01/01/2013 & $31 / 12 / 2017$ \\
\hline
\end{tabular}

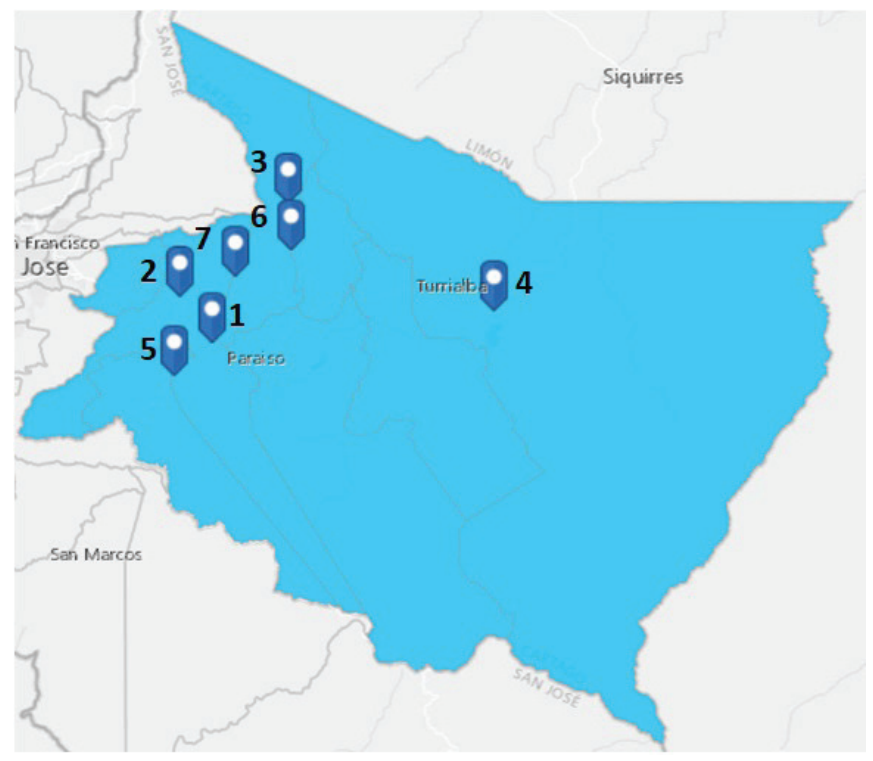

Figura 2. Ubicación de las estaciones meteorológicas según ID mostrado en el cuadro 1. 
Los datos son adquiridos con estaciones meteorológicas marca Campbell Scientific, que poseen anemómetros que mide la magnitud de la velocidad del viento en un rango de $0 \mathrm{~m} / \mathrm{s}$ a $50 \mathrm{~m} / \mathrm{s}$ con una precisión de $\pm 0,5 \mathrm{~m} / \mathrm{s}$ y un valor umbral de $0,5 \mathrm{~m} / \mathrm{s}$. Para la medición de la dirección del viento, disponen de una veleta que mide en un rango de $0^{\circ}$ a $360^{\circ}$ con una precisión de \pm $5^{\circ}$ y un valor umbral de $10^{\circ}$ para velocidades entre $0,8 \mathrm{~m} / \mathrm{s}$ y $1,8 \mathrm{~m} / \mathrm{s}$ y de $5^{\circ}$ para velocidades de $1,8 \mathrm{~m} / \mathrm{s}$ o mayores. También se utilizan estaciones meteorológicas marca Davis, equipadas con un anemómetro que mide magnitudes de la velocidad del viento en el rango de $0 \mathrm{~m} / \mathrm{s}$ a 80 $\mathrm{m} / \mathrm{s}$ con una precisión de $\pm 1 \mathrm{~m} / \mathrm{s}$, una resolución de $0,1 \mathrm{~m} / \mathrm{s}$ y un valor umbral de 0,4 m/s. La dirección del viento, se determina a través de una veleta que mide en un rango de $0^{\circ}$ a $360^{\circ}$ con una precisión de $\pm 3^{\circ}$ para valores de velocidad de 1,6 m/s o superiores.

\section{Descripción de los métodos de análisis}

Para caracterizar el recurso eólico se analizan los datos con el apoyo del software Matlab, mediante la escritura de códigos computacionales que permiten obtener resultados de la información colectada.

El primer análisis que se realiza es la representación de las series de tiempo de la velocidad del viento, posteriormente se grafica la velocidad del viento de forma mensual y horaria, para

visualizar variaciones estacionales y a lo largo de las 24 horas del día, respectivamente.

El segundo análisis que se efectúa es la distribución de frecuencia para las distintas velocidades del viento.

El tercer análisis que se efectúa es la intensidad de turbulencia, únicamente en la estación CIVCO, debido a su frecuencia de muestreo, que es cada 1 minuto.

Se utiliza la estadística descriptiva para poder representar, organizar y analizar la información recopilada de las estaciones meteorológicas. Este método permite identificar rangos o valores importantes; o patrones temporales del recurso eólico. Esto mediante medidas de tendencia central, en particular la media muestral, definida como: el promedio aritmético simple de los datos [27].

En el análisis de los datos se utiliza la media muestral de dos formas distintas, la primera para estimar distintos promedios del periodo de estudio, obteniendo datos horarios, diarios y mensuales. Con esto se tiene una descripción de la variación horaria y mensual del valor promedio del período comprendido por las estaciones meteorológicas. Los datos horarios, representan la variación del viento a lo largo de las 24 horas del día. Los datos mensuales permiten ver estacionalidad en los datos e identificar periodos de variación del viento a lo largo del año.

La segunda forma de utilizar la media muestral es para obtener los datos diarios y mensuales. De modo que se tiene una serie de tiempo diaria y mensual de los diez años de estudio, para cada estación meteorológica.

Se realizan histogramas de frecuencia, de modo que se representa gráficamente la distribución de frecuencia de los datos, se utiliza la frecuencia relativa.

También se utilizan medidas de dispersión, en particular la desviación estándar muestral, definida como la raíz cuadrada de la varianza muestral, es decir, de la medición de la distancia de los datos respecto a la media muestral. 
Tanto la media muestral como la desviación estándar muestral se utilizan para el cálculo de la intensidad de turbulencia $\left(I_{T}\right)$, una de las medidas básicas de la turbulencia del viento [28], dada por la ecuación (1).

$$
I_{T}=\frac{S}{\bar{x}}=\frac{\sqrt{\frac{\sum_{i=1}^{n}\left(x_{i}-\bar{x}\right)^{2}}{n-1}}}{\frac{1}{n} \sum_{i=1}^{n} x_{i}}
$$

Donde $S$ es la desviación estándar, $\bar{x}$ es el promedio de los datos, $i$ es el iésimo dato, $n$ es la cantidad total de datos y $x_{i}$ es el dato en la iésima posición.

Para el análisis de intensidad de turbulencia se toman los datos cada 1 minuto, disponibles únicamente en la estación CIVCO, se realiza mediante Matlab el procesamiento de los datos para generar promedios y desviaciones estándar cada 10 minutos. Posteriormente se eliminan todas las entradas que tienen velocidad cero, pues en este caso no es posible calcular la intensidad de turbulencia.

\section{Resultados y discusión}

A continuación, se presentan los resultados del análisis estadístico de la intensidad y dirección del viento en las provincias de Cartago. Para mostrar el comportamiento general del recurso eólico, en las figuras 3 y 4 se muestra la serie de tiempo de la magnitud de la velocidad del viento, en el período comprendido por cada estación. La figura 3 se refiere a las estaciones administradas por el ICE y muestra intensidades máximas entre los $5 \mathrm{~m} / \mathrm{s}$ y $7 \mathrm{~m} / \mathrm{s}$ en la estación de San Pablo de Cot; las menores intensidades están entre 0 m/s y $1 \mathrm{~m} / \mathrm{s}$ en la estación de Campamento. En el eje horizontal se ubica la escala temporal, se abarcan todos los datos de disponibles desde 2013 hasta 2018 y se coloca para orientación el mes de enero de cada año, indicado como "Jan".

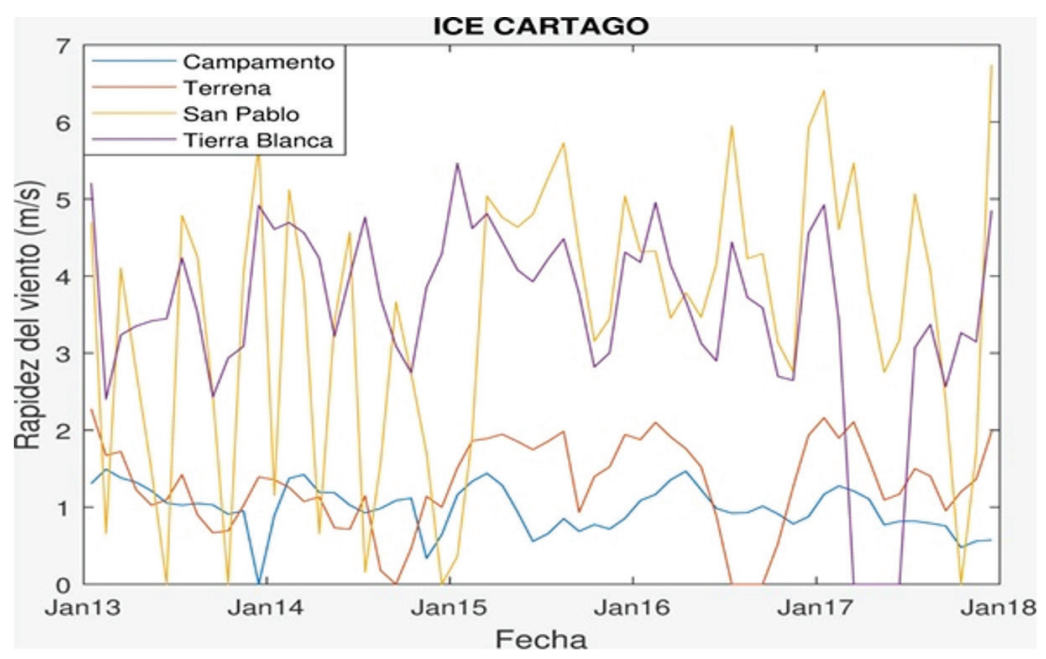

Figura 3. Series de tiempo de la magnitud del viento en las estaciones meteorológicas ubicadas en la provincia de Cartago, administradas por el ICE. 
La figura 4 se refiere a las estaciones administradas por el IMN y muestra intensidades máximas entre los $5 \mathrm{~m} / \mathrm{s}$ y $7 \mathrm{~m} / \mathrm{s}$ en la estación de RECOPE en Ochomogo; las menores intensidades están en la misma estación en periodos de calma $(0 \mathrm{~m} / \mathrm{s})$.

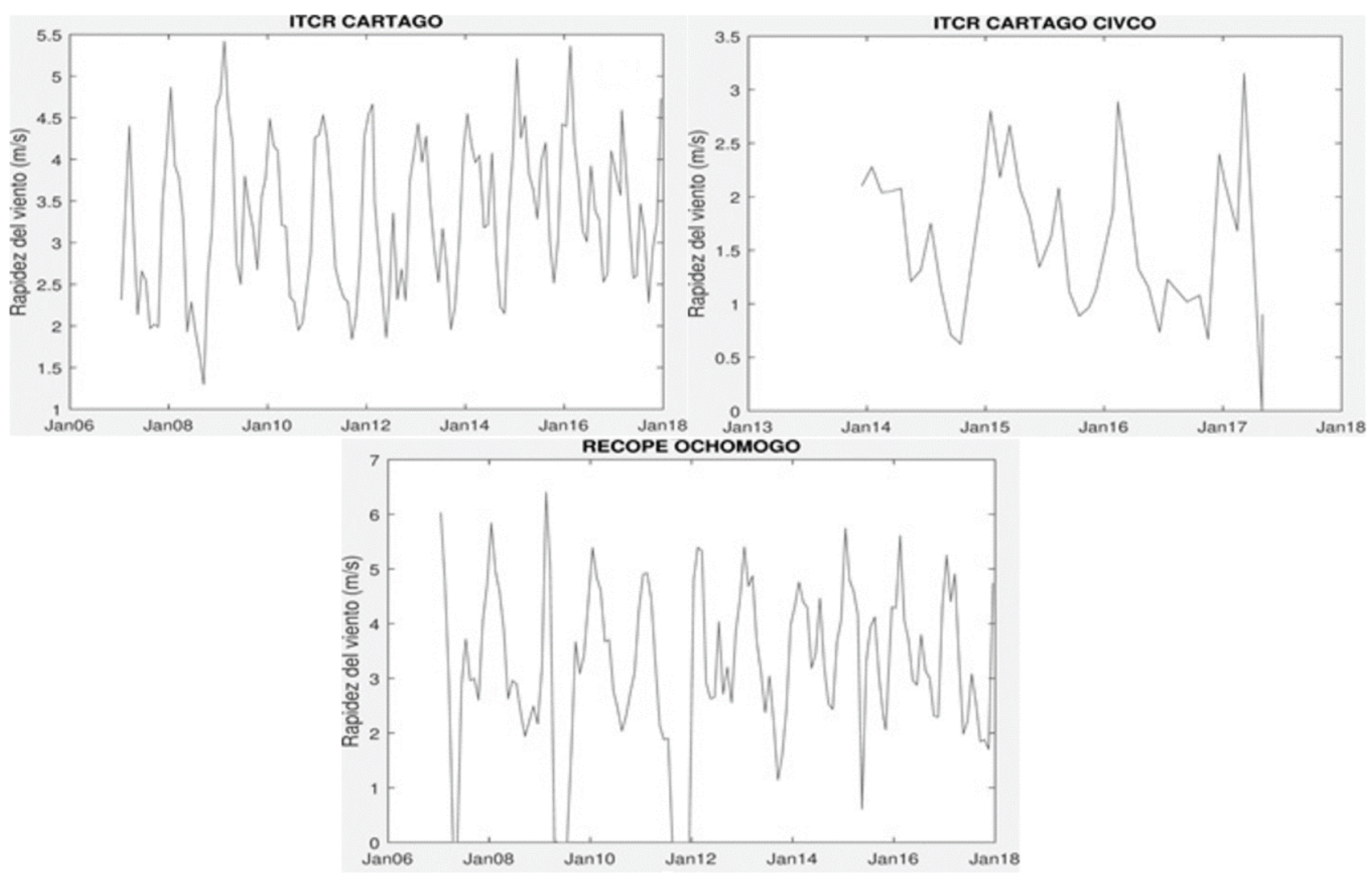

Figura 4. Series de tiempo de la magnitud de la velocidad del viento en las estaciones meteorológicas ubicadas en la provincia de Cartago, administradas por el IMN.

\section{Variación mensual del viento}

Al analizar los promedios anuales de todos los datos disponibles, se obtiene que para la región del valle central la variación mensual presenta un aumento del viento para los meses de fin de año e inicio de año, es decir los meses que van de noviembre hasta febrero. Luego se presenta una disminución de los datos con un pequeño aumento entre junio o julio, periodo conocido como las canículas; un mínimo se presenta en setiembre y octubre. En la figura 5 se presenta la distribución mensual de la magnitud de la velocidad del viento para las estaciones meteorológicas del estudio. Se muestra una distribución típica para la región climática. 

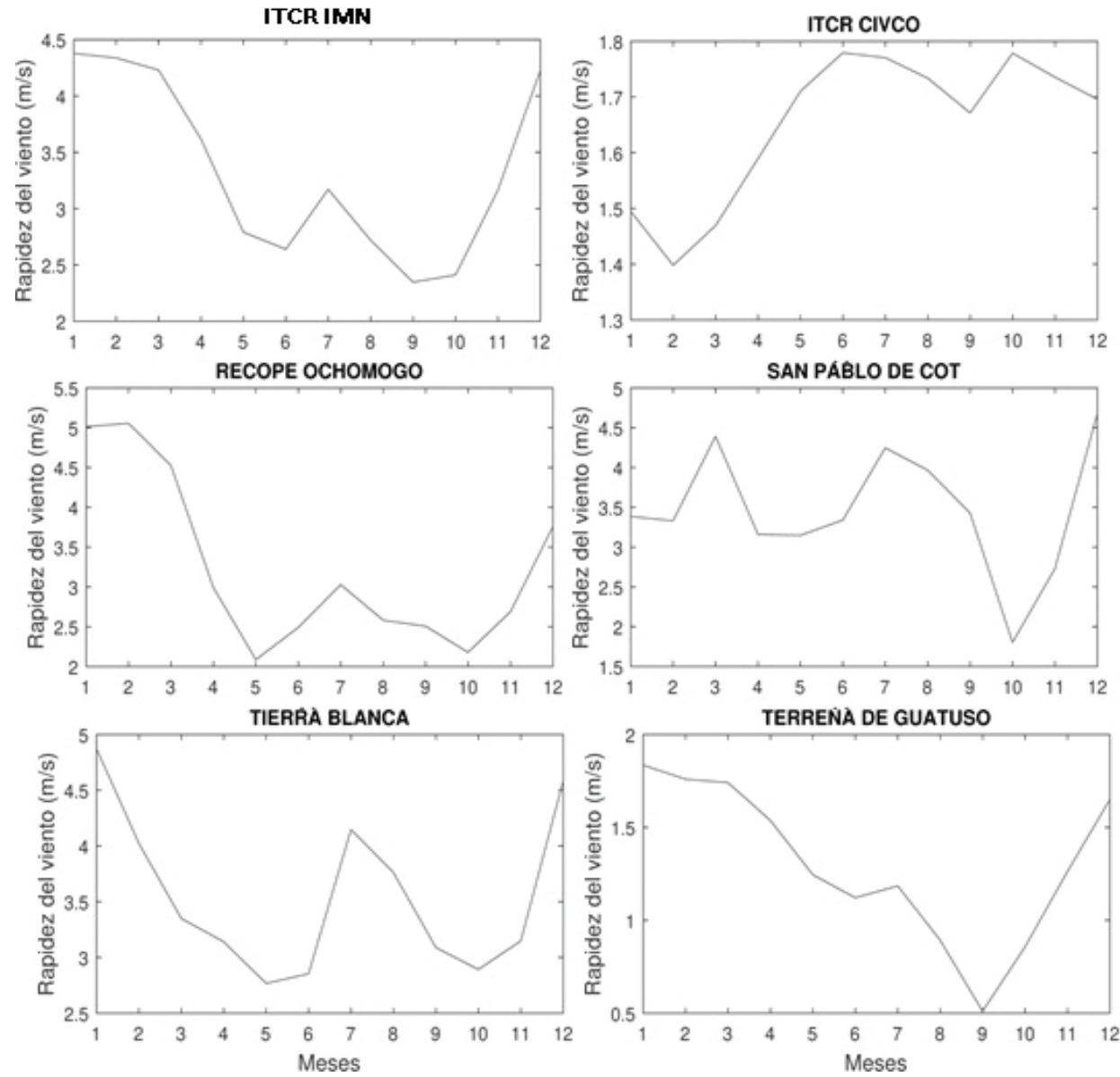

Figura 5. Distribución mensual de la magnitud del viento en las estaciones meteorológicas ubicadas en la provincia de Cartago (el eje horizontal indica el mes).

\section{Variación horaria del viento}

Utilizando las estaciones meteorológicas administradas por el IMN y el CIVCO, se logra ver la distribución a lo largo de las 24 horas del día. Donde los máximos de velocidad del viento se presentan cerca de mediodía, figura 6. Los máximos son entre $3 \mathrm{~m} / \mathrm{s}$ y $5 \mathrm{~m} / \mathrm{s}$ aproximadamente, la estación del CIVCO se encuentra a una altura menor que las estaciones del IMN, esa diferencia de altura se traduce en una diferencia en la magnitud del viento de $2 \mathrm{~m} / \mathrm{s}$ en el máximo y un desfase del máximo de dos horas aproximadamente, como se ve en la Figura 6. La estación del IMN ubicada en el Volcán Irazú presenta un comportamiento distinto, con intensidades casi constantes de $6 \mathrm{~m} / \mathrm{s}$ en horas de la noche y madrugada, con un decaimiento de la intensidad a partir de las 7:00 a.m. Ilegando al mínimo de 4 m/s, cerca de las 4:00 p.m., según se aprecia en la figura 6. 


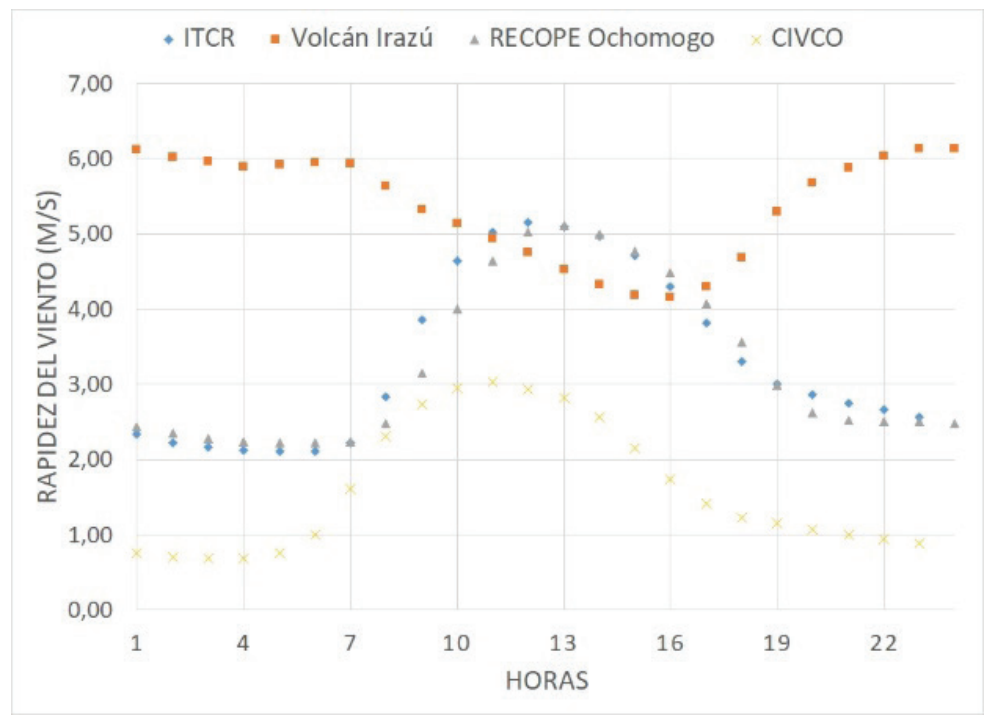

Figura 6. Distribución horaria de la magnitud del viento en las estaciones meteorológicas administradas por el IMN y el CIVCO.

\section{Dirección del Viento}

La provincia de Cartago se encuentra en la parte oriental del valle central y es influenciada por la Vertiente del Caribe, de modo que los vientos alisios son los que predominan en la provincia. En las figuras $7 \mathrm{a}-7 \mathrm{~d}$ se puede observar la rosa de los vientos para las estaciones meteorológicas administradas por el IMN y el ICE. En cada imagen se indica la escala de velocidad del viento en $\mathrm{m} / \mathrm{s}$.

Se puede determinar, según los datos de las estaciones, que las componentes predominantes son las Este o Norte, teniendo clara influencia los vientos alisios que entran por la Vertiente del Caribe y que afectan la provincia de Cartago.

ITCR CARTAGO

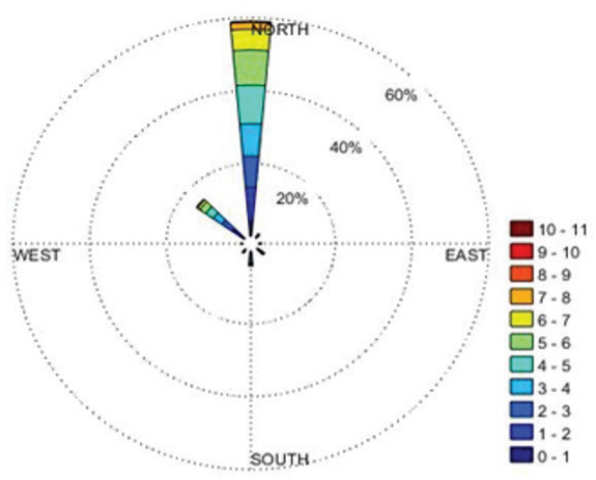

CIVCO ITCR CARTAGO

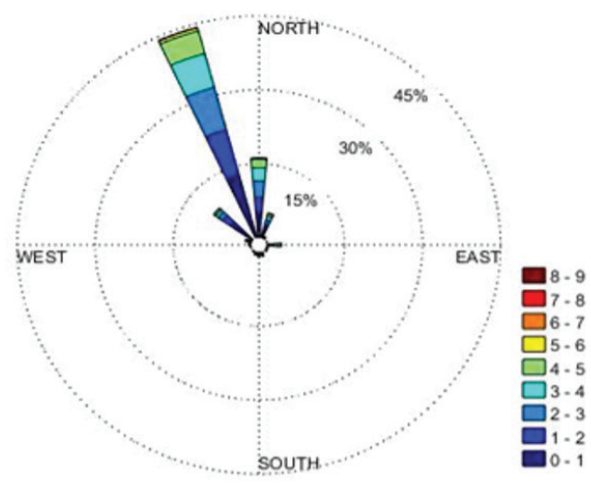

Figura 7a. Rosa de los vientos para las estaciones meteorológicas ITCR y CIVCO. 
RECOPE OCHOMOGO

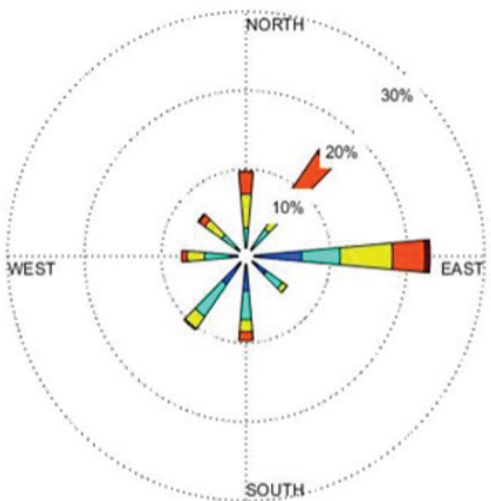

Figura 7b. Rosa de los vientos para las estaciones meteorológicas RECOPE Ochomogo y Volcán Irazú.

ICE CAMPAMENTO

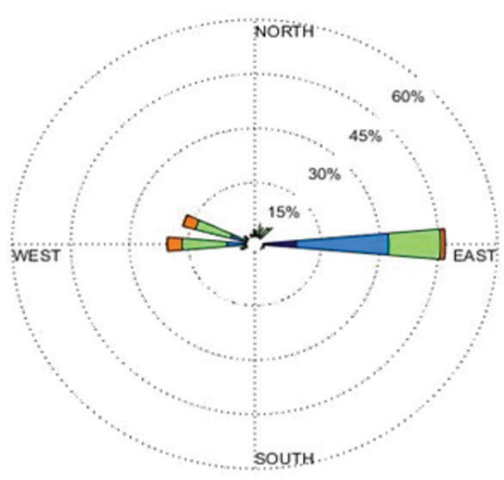

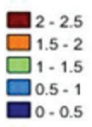

VOLCAN IRAZU CARTAGO

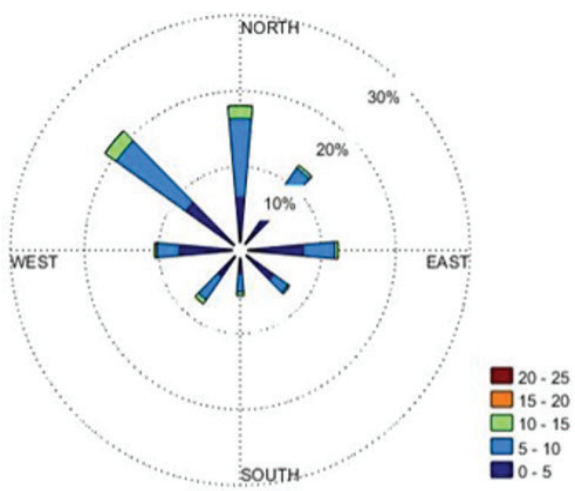

Figura 7c. Rosa de los vientos para las estaciones meteorológicas Campamento y San Pablo, administradas por el ICE.

ICE TIERRA BLANCA

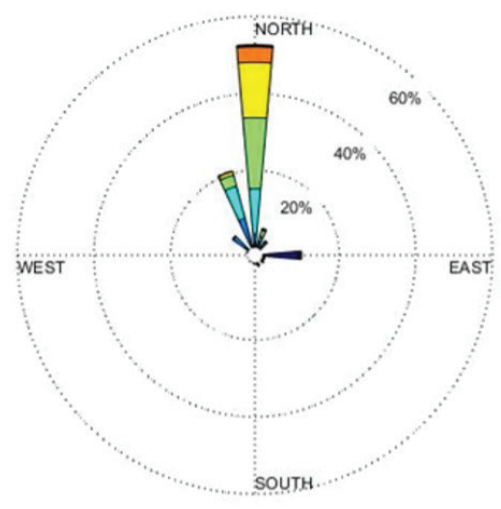

ICE TERRENA

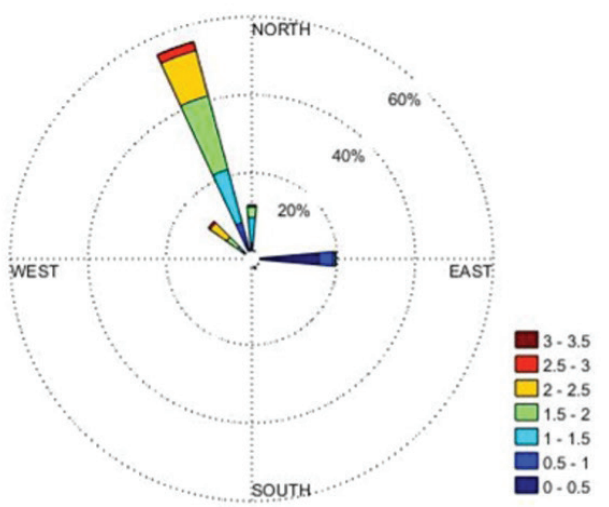

Figura 7d. Rosa de los vientos para las estaciones meteorológicas Tierra Blanca y Terrena, administradas por el ICE. 
Frecuencia de distribución de la velocidad del viento según su intensidad

Como se muestra en las figuras $8 \mathrm{a}$ y $8 \mathrm{~b}$, para la provincia de Cartago se cuenta con un predominio de las intensidades de velocidad de viento entre $1 \mathrm{~m} / \mathrm{s}$ y $2 \mathrm{~m} / \mathrm{s}$ aunque en dos de las estaciones la magnitud que predomina se encuentra entre $4 \mathrm{~m} / \mathrm{s}$ y $5 \mathrm{~m} / \mathrm{s}$, estas estaciones son las ubicadas en Tierra Blanca y en el volcán Irazú. Ambas estaciones se encuentran a mayor altitud y es esperable encontrar intensidades del viento mayores.

Es de mencionar que se consideró el viento calmo (intensidades de $0 \mathrm{~m} / \mathrm{s}$ ), representado por una barra gris en las figuras $8 \mathrm{a}$ y $8 \mathrm{~b}$, en tres de las ocho estaciones analizadas. El viento calmo representa el porcentaje mayor en la distribución de frecuencia en algunos casos, la estación donde esto es más evidente por la diferencia porcentual que se genera es en la estación administrada por el CIVCO, pero esta estación se encuentra a una altura sobre la superficie de 1,5 m esto hace que sea esperable que las intensidades del viento se encuentren dentro de las más bajas o que predomine el viento calmo.

La estación Volcán Irazú es la única que exhibe de forma clara la distribución típica de Weibull, que es característica de los histogramas de velocidad del viento.
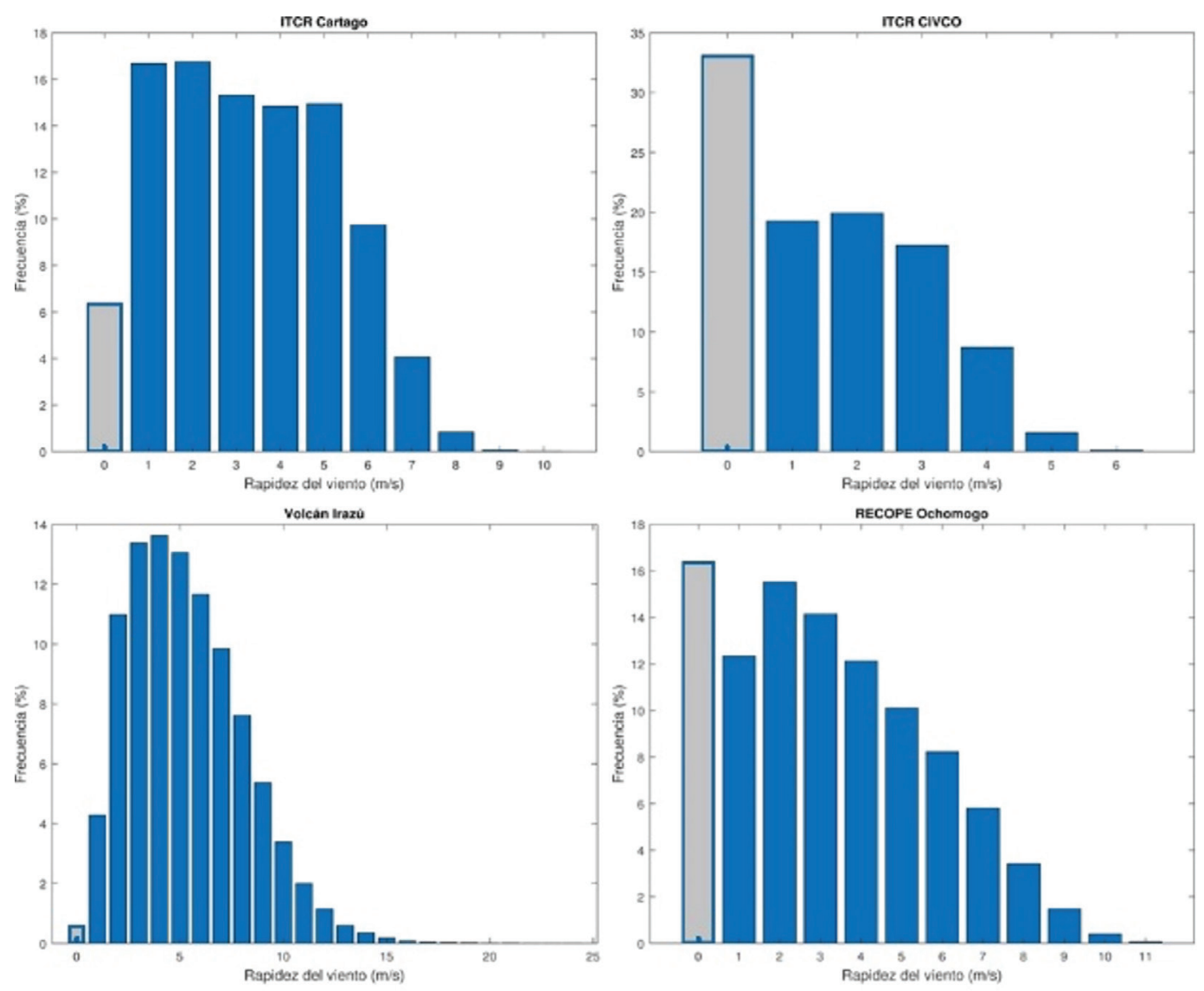

Figura 8a. Distribución de frecuencia de la magnitud de la velocidad del viento en las estaciones ITCR, CIVCO, Volcán Irazú y Ochomogo. 

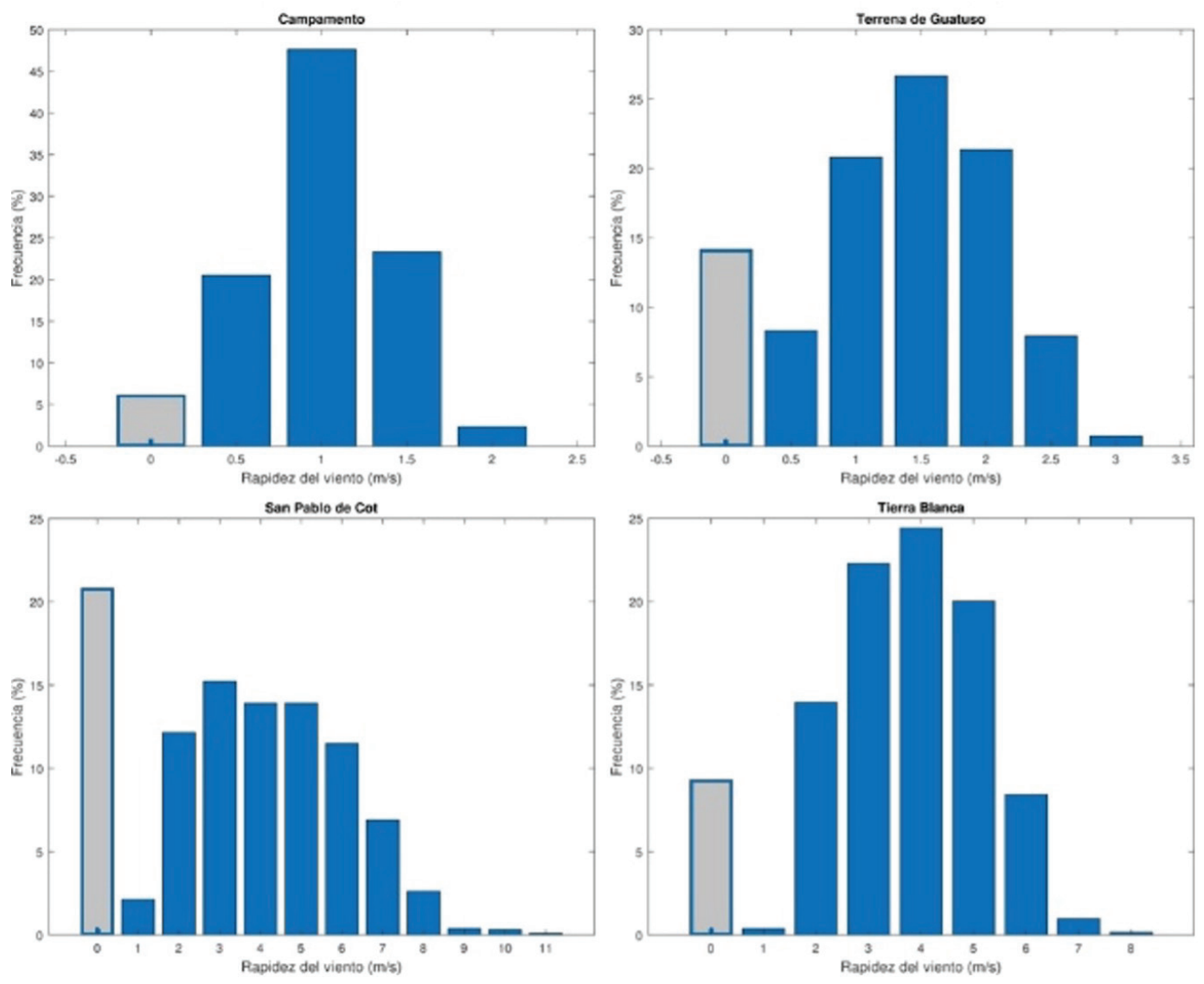

Figura $\mathbf{8 b}$. Distribución de frecuencia de la magnitud del viento en las estaciones Campamento, Terrena, San Pablo y Tierra Blanca.

\section{Intensidad de turbulencia en la estación CIVCO}

Al analizar los datos disponibles en la estación CIVCO, de un total de 151018 promedios tomados cada 10 minutos, se descartan 26796 promedios por ser iguales a cero.

Con los datos restantes se hace una primera vista de los datos y se obtienen valores de intensidad de turbulencia (IT) de hasta 300\%. Estos valores tan altos de IT se deben a que algunos promedios son muy cercanos a cero. Considerando la incertidumbre del equipo y la posible aplicación de la intensidad de turbulencia para diseño de turbinas eólicas, las cuales no operan a velocidades menores a $1 \mathrm{~m} / \mathrm{s}$ de velocidad de viento, se descartan todos los promedios menores a $1 \mathrm{~m} / \mathrm{s}$.

En este caso se descartan 38431 promedios, los cuales efectivamente corresponden a valores de IT entre $100 \%$ y $300 \%$ en su mayoría.

Siendo así, se descartaron en total 65 227, entre ceros y valores promedio menores a $1 \mathrm{~m} / \mathrm{s}$. Esto corresponde a un $43 \%$ de los datos disponibles, lo cual es previsible al ser una estación ubicada a 1,5 metros de altura sobre el nivel del suelo, como se mencionó anteriormente.

Con los datos restantes, un total de 85791 promedios calculados cada 10 minutos, se obtiene el histograma de la Figura 9, la única estación dentro de las que se incluyen en este estudio, que permite generar este tipo de gráficos debido a su frecuencia de muestreo. 


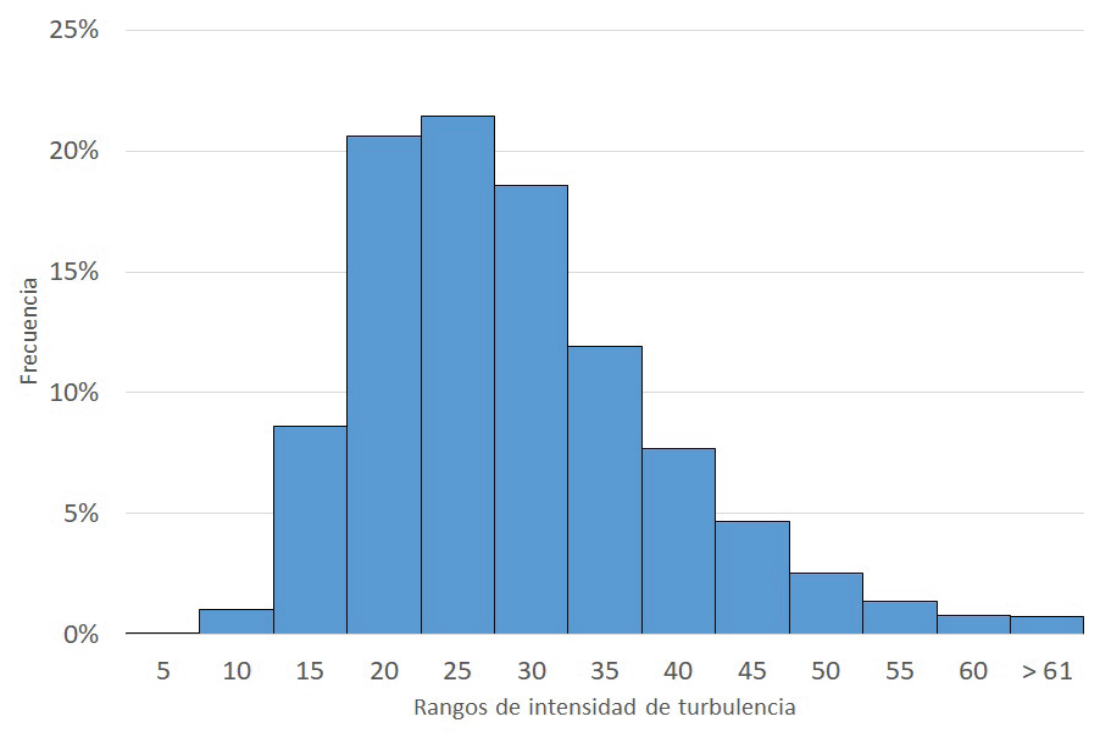

Figura 9. Histograma de la intensidad de turbulencia en la estación CIVCO.

En el histograma de la Figura 9, la cifra de intensidad de turbulencia en el eje horizontal, se interpreta como "menor o igual" para cada rango, siendo por ejemplo el número 10, el rango de IT mayor que 5\% y menor o igual que 10\%. De este modo las barras de mayor tamaño, indicadas como 20, 25 y 30, representan los valores de IT mayores que 15\% y hasta 30\%.

Estos rangos tan elevados de intensidad de turbulencia implican mucha variabilidad en la velocidad del viento. Se debe tener presente que los datos de esta estación se toman a 1,5 m sobre el nivel del suelo, por lo que es razonable registrar velocidades bajas y también capturar el efecto que tienen los obstáculos superficiales sobre el viento.

\section{Conclusiones}

Este estudio, el primero en su tipo en Costa Rica, orientado a analizar el recurso eólico en la provincia de Cartago, dentro de la capa límite superficial, con datos medidos a $10 \mathrm{~m}$ de altura sobre el nivel del suelo, permite indicar las siguientes características del recurso eólico en la provincia en estudio:

- En promedio los valores del viento en la región se encuentran entre los 3 y $5 \mathrm{~m} / \mathrm{s}$, esto a una altura de $10 \mathrm{~m}$ sobre la superficie.

- Al comparar dos estaciones ubicadas en el mismo punto, la estación a menor altura llega al máximo de velocidad de viento al menos 2 horas antes que la estación ubicada a 10 m de altura.

- La estación a mayor altura sobre el nivel del mar, pero siempre a $10 \mathrm{~m}$ sobre el nivel de la superficie, ubicada en el Volcán Irazú, registra las mayores velocidades en promedio, alrededor de $6 \mathrm{~m} / \mathrm{s}$.

- Con base en los datos tomados a 1,5 m de altura en las instalaciones del ITCR, la intensidad de turbulencia registra valores principalmente entre 15 y $30 \%$. 
En forma general, en la provincia de Cartago la magnitud del viento se puede diferenciar en dos periodos bien marcados, uno en los meses de fin e inicio de año (de noviembre a febrero) en los cuales las intensidades del viento son fuertes y otro periodo en los cuales la intensidad del viento disminuye hasta alcanzar los valores más bajos (septiembre y octubre).

Esta provincia presenta una fuerte influencia de los vientos alisios, provenientes de la Vertiente del Caribe, de modo que las direcciones que predominan son las componentes Norte o Este, siendo las dos componentes más recurrentes en los datos analizados.

\section{Referencias}

[1] International Renewable Energy Agency, «Transformación Energética Mundial, hoja de ruta hasta el 2050,» IRENA, Abu Dhabi, 2018.

[2] Global Wind Energy Council, «Global Wind Statistics,» Brussels, 2018.

[3] L. Merino, «Estado de la Nación 2017,» Servicios Gráficos AC, Pavas, 2017.

[4] L. M. Mora, «Programa País Carbono Neutralidad 2.0,» Dirección de Cambio Climático, 2017.

[5] R. D. Bolaños, «Desarrollo de los proyectos de energía eólica en Costa Rica (1979-2005),» Centro de Investigaciones Geofísicas, San Pedro, 2006.

[6] Ministerio de Ambiente y Energía, «Plan Nacional de Energía 2015-2030,» MINAE, San José, 2015.

[7] Instituto Costarricense de Electricidad, «Plan de expansión de la generación eléctrica 2016-2035,» Proceso de expansión del sistema de dirección de planificación desarrollo eléctrico, San José, 2017.

[8] Instituto Costarricense de Electricidad, «Plan de expansión de la generación eléctrica 2018-2034,» Proceso de expansión del sistema de la dirección de planificación y desarrollo eléctrico, San José, 2019.

[9] J. B. Morales, «Energías Renovables no Convencionales,» San José, 2015.

[10] T. Ackerman, F. Fernández, P. Schierhorn y F. Montoya, «Estudio de Red Costa Rica,» Energynautics, Darmstadt, 2017.

[11] J. De la cruz, G. Valencia y M. Vanegas, «Estudio estadístico de la velocidad y la dirección del viento en los departamentos de Atlántico y Bolívar en Colombia,» Ingeniare, vol. 26, n² 2, pp. 319-328, 2017.

[12] J. T. Millward-Hopkins, A. Tomlin, M. Pourkashanian, D. Ingham and L. Ma, «Mapping the Urban Wind Resource over UK Cities using an Analytical Downscaling Method,» Copenhagen, 2012.

[13] P. Enevoldsen and F. Hendrik «Mapping the Wind Energy Potential of Sweden: A Sociotechnical Wind Atlas,» Hindawi, p. 11, 2018.

[14] D. A. Fadare, «The application of artificial neural networks to mapping of wind speed profile for energy application in Nigeria,» Applied Energy, vol. 3, n 87, pp. 934-942, 2010.

[15] K. Ono and T. Uchida, «High-performance parallel simulation of airflow for complex terrain surface,» Hindawi, pp. 1-10, 2019.

[16] D. Elliott, «Wind Resource Assessment and mapping for Afghanistan and Pakistan,» National Renewable Energy Laboratory, Colorado, 2011.

[17] M. Landry, Y. Ouedraogo, Y Gagnon and A. Ouedraogo, "On the wind resource mapping of Burkina Faso," International Journal of Green Energy, vol. 2, n 14, pp. 150-156, 2017.

[18] A. Prasad, R. A. Taylor and M. Kay, «Assessment of solar and wind resource synergy in Australia,» Applied Energy, pp. 354-367, 2017.

[19] L. Li, X. Wang , L. Luo, Y. Zhao, X. Zong and N. Bachagha, « Mapping of wind energy potential over the Gobi Desert in Northwest China based on multiple sources of data,» Frontiers of Earth Science, vol. 2, n 12, pp. 264-279, 2018.

[20] B. R Furevik, A. M. Sempreviva, L. Cavaleri, J. M Lefèvre and C. Transerici, «Eight years of wind measurements from scatterometer for wind resource mapping in the Mediterranean Sea,» Wind Energy, vol. 3, n 14, pp. 355372, 2011.

[21] C. Jung and D. Schindler, «3D statistical mapping of Germany's wind resource using WSWS,» Energy Conversion and Management, $\mathrm{n}^{\circ}$ 159, pp. 96-108, 2018.

[22] L. Muñoz, «Decimoquinto informe Estado de la Nación en Desarrollo Humano Sostenible» Pavas, 2008. 
[23] M. Brower, J. W. Zack, B. Bailey, M. N Schwartz and D. L. Elliott «Mesoscale modeling as a tool for wind resource assessment and mapping,» National Renewable Energy Laboratory, Colorado, 2004.

[24] Instituto de Desarrollo Rural, «Región Central Caracterización del Territorio Cartago-Oreamuno-El Guarco-La Unión,» 2016.

[25] Instituto Meteorológico Nacional, «El clima, su variabilidad y cambio climático en Costa Rica, San José, 2008.

[26] R. Villalobos y J. Solano, «Regiones y subregiones climáticas de Costa Rica,» 2012. 